\title{
Continuous Preview Control of Dual-Stage Actuator Systems for Reduced Transition Time
}

\author{
Aurélio Salton, Zhiyong Chen, Jinchuan Zheng and Minyue Fu
}

\begin{abstract}
Preview Control design is proposed in order to reduce the settling time of Dual-Stage Actuators (DSA's). It is shown that a significantly better performance is achieved by exploring the extra degree of freedom provided by the secondary actuator before the output transition instant. Given the information of the immediate future reference point, the proposed strategy allows the slow actuator to move ahead of time while the fast actuator maintains the total output at the desired reference. Experimental results demonstrate the effectiveness of the proposed methodology when compared to a conventional form of DSA control design.
\end{abstract}

\section{INTRODUCTION}

In this paper we address the issue of reducing the output transition time interval of Dual-Stage Actuators (DSA's) from an initial reference $\operatorname{ref}(t \leq \tau)=\operatorname{ref}_{i}$ to a final reference $\operatorname{ref}(t>\tau)=\operatorname{ref}_{i+1}$. The proposed approach makes use of the information on future reference points in order to compute a pair of inputs to be applied before the transition instant $(t=\tau)$. This preview control strategy improves the performance of any system that accounts with redundant actuators and prior knowledge of future reference points, and is particularly interesting for systems facing the successive set point scenario.

The class of systems to be considered are Dual-Stage Actuators such as dual-stage Hard-Disk Drives (HDDs) [1]. These systems are defined as two actuators connected in series, a primary (slow) actuator, responsible for providing the system with a long range, and a secondary (fast) actuator, responsible for improving the accuracy and speed of the system. The primary actuator is considered a rigid body of mass $M$ and it is assumed that the friction acting on this actuator (if any) is actively compensated by a friction compensator. The secondary actuator is treated as a body of mass $m$ connected to a spring of constant $k$ with damping $c$. The secondary actuator is connected in series with the primary and has a range of actuation bounded by $\pm r(r>0)$. Typically, DSA's have the features that $M \gg m, y_{1} / y_{2} \gg 1$, and $\left|u_{2} / u_{1}\right| \gg m / M$, thus, the coupling forces between the primary and the secondary actuators may be neglected for simplicity.

The authors are with the School of Electrical Engineering and Computer Science, The University of Newcastle, Callaghan, NSW 2308, Australia. This work was supported by the Australian Research Councils Center of Excellence for Complex Dynamic Systems and Control (CDSC). e-mail: c3085114@uon.edu.au; \{Zhiyong.Chen, Jinchuan.Zheng, Minyue.Fu\}@newcastle.edu.au.
In this way, the DSA of interest is modeled as a linear decoupled dual-input single-output (DISO) system, which is represented in a state-space form as:

$$
\begin{array}{r}
\Sigma_{1}: \dot{x}_{1}=A_{1} x_{1}+B_{1} u_{1}, x_{1}(0)=0,\left|u_{1}\right| \leq \bar{u}_{1} \\
\Sigma_{2}: \dot{x}_{2}=A_{2} x_{2}+B_{2} u_{2}, x_{2}(0)=0,\left|u_{2}\right| \leq \bar{u}_{2} \\
y=y_{1}+y_{2}=C_{1} x_{1}+C_{2} x_{2},
\end{array}
$$

where $x_{1}=\left[\begin{array}{ll}y_{1} & \dot{y}_{1}\end{array}\right]^{\top}$ is associated with the primary (coarse) actuator and $x_{2}=\left[\begin{array}{ll}y_{2} & \dot{y}_{2}\end{array}\right]^{\top}$ with the secondary (fine) actuator, and $\bar{u}_{i}$ is the control saturation level for $u_{i}$. Furthermore,

$$
\begin{gathered}
A_{1}=\left[\begin{array}{ll}
0 & 1 \\
0 & 0
\end{array}\right], B_{1}=\left[\begin{array}{c}
0 \\
b_{1}
\end{array}\right], C_{1}=\left[\begin{array}{ll}
1 & 0
\end{array}\right], \\
A_{2}=\left[\begin{array}{cc}
0 & 1 \\
a_{1} & a_{2}
\end{array}\right], B_{2}=\left[\begin{array}{c}
0 \\
b_{2}
\end{array}\right], C_{2}=\left[\begin{array}{ll}
1 & 0
\end{array}\right],
\end{gathered}
$$

with $a_{1}=-k / m, a_{2}=-c / m, b_{1}=1 / M$ and $b_{2}=1 / m$.

As opposed to DSA control design, a vast number of important achievements regarding settling time reduction can be found in the literature for single stage systems. While Time Optimal Control (TOC) performance is still not achievable without the problems related to chattering and robustness, the past few decades have provided us with a number of controllers that are able to approximate TOC performance. More specifically, an important adaptation of TOC was proposed by Workman [3] under the name of proximate timeoptimal servomechanism (PTOS). This control law enables to system to avoid problems related to chattering inasmuch as it only uses the maximal acceleration of the actuator when it is practical to do so: as the system approaches the reference point the controller switches to a linear feedback control gain. More recently, a different approach was presented by Lin et al. [4], and then Chen et al. [5] where the composite nonlinear feedback (CNF) was developed. This controller is able to achieve an improved performance without increasing the system bandwidth, once it uses dynamic damping to do so. Unfortunately, neither these control strategies are readily adaptable to DSA's nor make fully use of the redundancy of actuation.

The first attempt to integrate and adapt both the CNF and PTOS to DSA's was proposed by Zheng et al. [6]. This combined controller improves the overall performance of the system while accommodating the saturation of the secondary actuator. The PTOS control law is applied to the primary actuator so that it yields a closed-loop system with a fast rise time and certain allowable overshoot; the CNF control law is applied to the secondary actuator in 
order to eliminate the overshoot generated by the primary. Conversely, conventional work on DSA tracking control is based on designing a primary actuator control loop to yield a small or no overshoot, and a secondary actuator controller to follow the position error of the primary [8] and [9]. While the association of PTOS and CNF generates a significantly better performance, both the conventional and the nonlinear strategies do not take into account the possibility of preactuation.

Due to the redundancy of actuators, dual-stage systems present liberty of movement outside the transition time interval, i.e., outside the time interval when the system output is moving from one reference point to another. As shown in Fig. 1 , we take advantage of this extra degree of freedom provided by the secondary actuator and allow the primary actuator to move ahead of time [7]. The main contribution of the paper is the development of this pre-actuation strategy based on the immediate future reference point in order to reduce the settling time of the system. Moreover, it is shown that the proposed preview control methodology is fully compatible with the nonlinear state-of-art DSA design proposed in [6]. In fact, a continuous switching between both controllers is achieved for the primary actuator. Experimental results demonstrate the effectiveness of the proposed design, which can achieve an improvement of up to $75 \%$ regarding the settling time when compared to a conventional form of DSA control.

As opposed to the problem of pure settling time reduction, the minimum time/energy output-transition problem was already studied in the preview control framework in [10], where a form of pre-actuation is allowed. This technique, however, is not used to solely reduce the settling time of the system and does not consider the saturation of the secondary actuator, which is a hard constraint in the design of fast tracking controllers for DSA's.

To present the proposed methodology, the paper is divided into the following sections: Section II formulates the problem and introduces the general idea, the proposed solution is given in Section III, experimental results are presented in Section IV, and Section V concludes the paper.

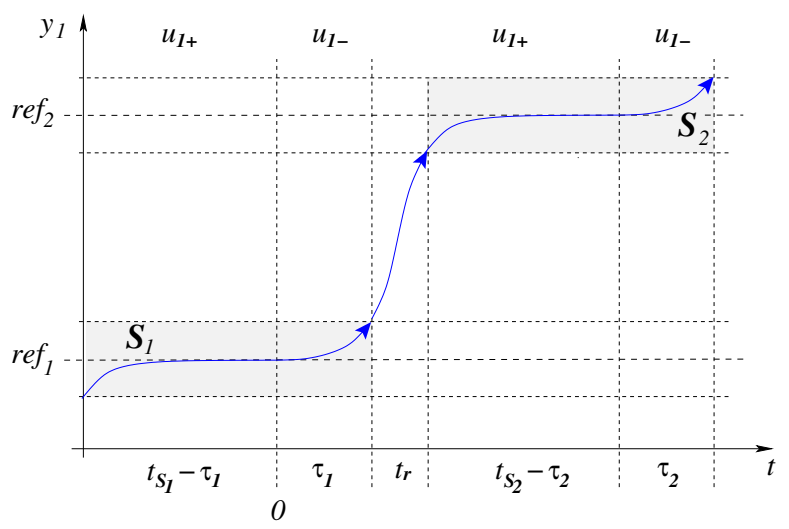

Fig. 1. Schematic representation of the Preview Control Strategy: after $t_{S_{1}}$ seconds in $S_{1}, y_{1}$ must move to $S_{2}$ with reduced transition time $t_{r}$.

\section{PROBlem Formulation}

An intrinsic characteristic of DSAs is that the coarse and fine actuators are complementary to each other: while the primary actuator is slow and has a large travel range, the secondary actuator is fast but has a limited range of actuation. Due to these complementary characteristics of the DSA it will be assumed that, within the travel range of the secondary actuator, the tracking error of the primary actuator is sufficiently smooth to be compensated with negligible error.

In other words, if we define a manifold $\boldsymbol{S}_{i}$,

$$
\boldsymbol{S}_{i}=\left\{y_{1} \in \mathbb{R}:\left|y_{1}-\operatorname{ref}_{i}\right| \leq r\right\},
$$

where $\operatorname{ref}_{i}$ is the $i$-th reference point and $\pm r$ is the range of the secondary actuator, then, whenever $y_{1}$ is within the manifold $\boldsymbol{S}_{i}$, the total output of the system $y$ will be at the $i$-th reference point with negligible error due to the actions of the secondary actuator. Moreover, if the output $y$ must stay at an initial reference point $\operatorname{ref}_{1}$ for $t_{S}$ seconds before moving to another given reference ref $_{2}$, then $y_{1}$ must stay in $S_{1}$ for $t_{S}$ seconds before moving to $S_{2}$ (Fig. 1).

Therefore, we will address each actuator control design separately as follows.

\section{A. Primary actuator control problem:}

For a given initial condition $x_{1}(0)=\left[\begin{array}{ll}\operatorname{ref}_{1} & 0\end{array}\right]^{\top}$, two manifolds $\boldsymbol{S}_{1}$ and $\boldsymbol{S}_{2}$ determined by (2), and a control saturation level $\bar{u}_{1}$, find a controller

$$
\left|u_{1}(t)\right| \leq \bar{u}_{1}, \quad t \geq 0
$$

and a preview control time $\tau \geq 0$, such that, the output $y_{1}$ of the primary actuator is driven from $S_{1}$ to $S_{2}$ with a reduced transition time $t_{r}$, in the following sense:

$$
\begin{aligned}
& y_{1}(t) \in \boldsymbol{S}_{1}, 0 \leq t \leq \tau . \\
& y_{1}(t) \in \boldsymbol{S}_{2}, t \geq \tau+t_{r}, \text { and } \lim _{t \rightarrow \infty} y_{1}(t)=\operatorname{ref}_{2} .
\end{aligned}
$$

\section{B. Secondary actuator control problem:}

For a control saturation level $\bar{u}_{2}$, find a controller

$$
\left|u_{2}(t)\right| \leq \bar{u}_{2}, \quad t \geq 0
$$

for the secondary actuator to compensate for the error generated by the primary actuator, i.e., to achieve $y=y_{1}+y_{2}=$ $\operatorname{ref}_{i}$ when $y_{1} \in \boldsymbol{S}_{i}$.

Remark 2.1: Notice that if one chooses $\tau=0$ one falls in the conventional control strategy for DSA where no preview control is applied. Conversely, there must be an upper bound in the preview control time inasmuch as $t_{S}-\tau$ must be long enough such that the primary actuator can be driven sufficiently close to the reference before any pre-actuation is applied. 


\section{Proposed Solution}

In order to solve problem II-A the proposed control strategy will be composed by two different control laws, i.e.,

$$
u_{1}(t)=\left\{\begin{array}{ll}
u_{1-}(t), & 0 \leq t \leq \tau \\
u_{1+}(t), & t>\tau
\end{array} .\right.
$$

In particular, 1) a preview control law, denoted by $u_{1-}$, will be responsible for the pre-actuation of the system; 2) a nonlinear feedback control law, denoted by $u_{1+}$, will be applied in order to achieve $y_{1} \rightarrow \operatorname{ref}_{2}$, as $t \rightarrow \infty$. Practically, we can assume that $y_{1}$ approaches ref $_{2}$ in a short time (not necessarily $t \rightarrow \infty$ ), say in $\tau_{1}+t_{r}+t_{S_{2}}-\tau_{2}$ as denoted in Fig. 1. More specifically, the primary actuator's status becomes $\left[\begin{array}{lll}\mathrm{ref}_{2} & 0\end{array}\right]^{\top}$ at this moment. Taking this moment as the new reference time $t=0$, the proposed controller (7) may apply recursively in a successive step tracking scenario. Problem II-B will be solved by a single composite nonlinear control law that allows the secondary actuator to compensate for the error generated by the primary both during $u_{1-}$ and $u_{1+}$. Next, we will study the solvability of II-A and II-A respectively with the emphasis on the former.

\section{A. Solvability of II-A}

In order to simplify the notation, let us define

$$
p:=y_{1}, \quad v:=\dot{y}_{1},
$$

so that $x_{1}=\left[\begin{array}{ll}p & v\end{array}\right]^{\top}$ and the primary actuator equation becomes

$$
\dot{p}=v, \quad \dot{v}=u_{1} / M .
$$

1) PTOS Controller $u_{1+}$ : In literature, e.g., [9], a well known proximate time-optimal servomechanism (PTOS) control is applied to the primary actuator. This is a near time-optimal control strategy that can accommodate plant uncertainty, measurement noise and actuator saturation. The control law is given by:

$$
u_{1+}(t)=\operatorname{sat}\left[\sigma(t) f\left(\operatorname{ref}_{2}-p(t)\right)-k_{2} v(t)\right]
$$

where the non-negative continuous function $f$ is defined as

$$
f(x)=\left\{\begin{array}{ll}
k_{1}|x|, & \text { for }|x| \leq \bar{u}_{1} / k_{1} \\
2 \sqrt{k_{1} \alpha|x| \bar{u}_{1}}-\bar{u}_{1}, & \text { for }|x|>\bar{u}_{1} / k_{1}
\end{array},\right.
$$

$\sigma(t)=\operatorname{sgn}\left(\operatorname{ref}_{2}-p(t)\right), 0 \leq \alpha \leq 1$ and $\operatorname{sat}(\cdot)$ is with the saturation level of $\bar{u}_{1}$. The constants $k_{1}$ and $k_{2}$ are positive and they can be designed by any linear control technique, e.g., pole-placement method. The role of the PTOS controller (9) can be summarized as follows for the need of this paper.

Lemma 3.1: For given parameters $\tau, p_{\tau}, v_{\tau}, \operatorname{ref}_{2}$ and $\boldsymbol{S}_{2}$ determined by (2), the controller (9) with $t \in[\tau, \infty)$ drives the primary actuator from $x_{1}(\tau)=\left[p_{\tau} v_{\tau}\right]^{\top}$ into $\mathbf{S}_{2}$ in the sense of (5). In particular, the transition time $t_{r}$ is called PTOS-optimized w.r.t. $\left(v_{\tau}\right.$, ref $\left._{2}-p_{\tau}\right)$.

The main objective of this paper is to reduce the transition time $t_{r}$. Clearly, for a given system and a given PTOS controller, $t_{r}$ depends on the initial velocity $v_{\tau}$ and the initial step level $\mathrm{ref}_{2}-p_{\tau}$. Without loss of generality, we assume ref $_{2}>$ ref $_{1}$. Roughly speaking, $t_{r}$ is reduced if $v_{\tau}$ is larger and ref $_{2}-p_{\tau}$ is smaller. In a conventional control design, the PTOS controller applies with $v_{\tau}=0$ and $p_{\tau}=\operatorname{ref}_{1}$. In other words, $t_{r}$ is PTOS-optimized w.r.t. $\left(0, \mathrm{ref}_{2}-\mathrm{ref}_{1}\right)$. In this paper, a preview controller is introduced such that $t_{r}$ is PTOS-optimized w.r.t. $\left(v_{\tau}, \mathrm{ref}_{2}-\mathrm{ref}_{1}-r\right)$ for some $v_{\tau}>0$.

2) Preview Controller $u_{1-}$ : The preview control strategy is based on the knowledge that after $t_{S}$ seconds at $\mathrm{ref}_{1}$ the total output $y$ will move to ref $_{2}$. As already mentioned in Section II, two manifolds $\boldsymbol{S}_{1}$ and $\boldsymbol{S}_{2}$ are built around the respective references, and the problem, in the primary actuator perspective, becomes to move from $S_{1}$ to $S_{2}$ while satisfying the constraints (3) and (4). In order to reduce the transition time from one manifold to another, a pre-actuation will be applied to the primary actuator such that at the transition instant $t_{S}$ the output $y_{1}$ is at the border of $\boldsymbol{S}_{1}$ moving toward $S_{2}$ (Fig. 1). We assume the preview control time is defined as $\tau$, i.e., the pre-actuation starts $\tau$ seconds before the transition instant. In this context, a trajectory must be designed such that the primary actuator changes from a given initial condition $x_{1}(0)=\left[\begin{array}{ll}p_{0} & 0\end{array}\right]^{\top}$ to a desired condition $x_{1}(\tau)=\left[\begin{array}{ll}p_{\tau} & v_{\tau}\end{array}\right]^{\top}$. Specifically, $p_{0}=\operatorname{ref}_{1}$ and $p_{\tau}=\operatorname{ref}_{1} \pm r$ in the present scenario. Among many possible trajectories, the one with a minimum input effort is selected.

Lemma 3.2: For any given $\tau>0, p_{0}, p_{\tau}$, and $v_{\tau}$, the minimum effort input that takes the primary actuator from the initial condition $x_{1}(0)=\left[\begin{array}{ll}p_{0} & 0\end{array}\right]^{\top}$ to the final condition $x_{1}(\tau)=\left[p_{\tau} v_{\tau}\right]^{\top}$, is given by:

$$
\begin{array}{r}
u_{1-}(t)=M(a t+b), \quad 0 \leq t \leq \tau \\
a=6\left(\tau v_{\tau}-2 \Delta p\right) / \tau^{3}, b=-2\left(\tau v_{\tau}-3 \Delta p\right) / \tau^{2}
\end{array}
$$

where $\Delta p:=p_{\tau}-p_{0}$.

Proof: This follows from standard calculus of variations in optimal control theory. For a detailed proof please refer to [2].

The controller (10) generates a smooth trajectory for the primary actuator while taking it from initial conditions $x_{1}(0)=\left[\begin{array}{ll}p_{0} & 0\end{array}\right]^{\top}$ to final conditions $x_{1}(\tau)=\left[\begin{array}{ll}p_{\tau} & v_{\tau}\end{array}\right]^{\top}$. This controller, however, does not always satisfy the constraints (3) - (5). Moreover, at the instants $t=0$ and $t=t_{S}$ there will be switchings from the PTOS controller $\left(u_{1+}\right)$ to preview controller $\left(u_{1-}\right)$ and vice verse, which may cause discontinuity in the overall controller (7). Theorem 3.1 shows that with a proper choice of the preview time $\tau$ and the final velocity $v_{\tau}$, the controller (10) not only satisfies the constraints but also provides a continuous switching between the preview controller and the PTOS controller.

Theorem 3.1: For any given $r>0, \operatorname{ref}_{i}$ and $\mathbf{S}_{i}$ determined by (2), $i=1,2$, let $\delta:=\operatorname{ref}_{2}-\operatorname{ref}_{1}, \sigma=\operatorname{sgn}(\delta)$, and $\xi=\delta-\sigma r$, and assume $|\delta| \geq 2 r$. Consider a primary actuator with the initial condition $x_{1}(0)=\left[\mathrm{ref}_{1}, 0\right]$.

(i) For any $\tau$ and $v_{\tau}$ satisfying $\tau>0$ and $\sigma v_{\tau}>0$, the controller (7) composed of (9) and (10) with $p_{0}=$ ref $_{1}$ and $p_{\tau}=\operatorname{ref}_{1}+\sigma r$ drives the primary actuator from $\mathbf{S}_{1}$ 
to $\mathbf{S}_{2}$ in the sense of (5). Moreover, the transition time $t_{r}$ is PTOS-optimized w.r.t. $\left(v_{\tau}, \xi\right)$.

(ii) In (i), if $\tau$ and $v_{\tau}$ satisfy

$$
\tau v_{\tau}=3 \sigma r, v_{\tau}=\sigma \sqrt{\rho \operatorname{sat}\left(\bar{v}_{\tau}^{2} / \rho\right)}
$$

where

$$
\bar{v}_{\tau}=\sqrt{\left(\rho k_{2} / 2\right)^{2}+\rho f(\xi)}-\rho k_{2} / 2, \rho:=3 r /(2 M),
$$

the controller (7) is continuous over $[0, \infty)$ and the constrains (3) and (4) are satisfied. Thus, the problem II-A is solved.

Proof: (i) During the time interval $[0, \tau)$, Lemma 3.2 shows that the primary actuator is driven by the controller (10) from $x_{1}(0)=\left[\begin{array}{ll}p_{0} & 0\end{array}\right]^{\top}$ to $x_{1}(\tau)=\left[\begin{array}{ll}p_{\tau} & v_{\tau}\end{array}\right]^{\top}$. During $[\tau, \infty)$, the initial velocity for the PTOS controller (9) is $v_{\tau}$ and the initial step level is $\xi$. Obviously, the transition time $t_{r}$ is PTOS-optimized w.r.t. $\left(v_{\tau}, \xi\right)$ by Lemma 3.1.

(ii) Because the first equation of (11) implies $b=0$ in (10) by noting $p_{\tau}-p_{0}=\sigma r$, the controller (10) becomes $u_{1-}(t)=$ Mat and hence $u_{1-}(0)=0$.

At the time $\tau$, we have

$$
\begin{gathered}
u_{1-}(\tau)=M a \tau=\sigma v_{\tau}^{2} / \rho \\
u_{1+}(\tau)=\operatorname{sat}\left(\sigma f(\xi)-k_{2} v_{\tau}\right) .
\end{gathered}
$$

To show $u_{1+}(\tau)=u_{1-}(\tau)$, we consider two cases.

(a) If $|\xi|$ and hence $f(\xi)$ is large such that $\bar{v}_{\tau}^{2} \geq \rho \bar{u}_{1}$. From the second equation of (11), we have $v_{\tau}=\sigma \sqrt{\rho \bar{u}_{1}}$. As a result, on one hand, (12) gives

$$
u_{1-}(\tau)=\sigma \bar{u}_{1}
$$

on the other hand, (13) gives

$$
u_{1+}(\tau)=\sigma \operatorname{sat}\left(f(\xi)-k_{2} \sqrt{\rho \bar{u}_{1}}\right) .
$$

It suffices to show

$$
f(\xi)-k_{2} \sqrt{\rho \bar{u}_{1}} \geq \bar{u}_{1}
$$

to prove $u_{1+}(\tau)=u_{1-}(\tau)$. Indeed, $\bar{v}_{\tau} \geq \sqrt{\rho \bar{u}_{1}}$ gives

$$
\sqrt{\left(\rho k_{2} / 2\right)^{2}+\rho f(\xi)} \geq \rho k_{2} / 2+\sqrt{\rho \bar{u}_{1}}
$$

and hence (14).

(b) If $|\xi|$ and hence $f(\xi)$ is small such that $\bar{v}_{\tau}^{2}<\rho \bar{u}_{1}$. From the second equation of (11), we have $v_{\tau}=\sigma \bar{v}_{\tau}$. Then, (12) gives

$$
u_{1-}(\tau)=\sigma \bar{v}_{\tau}^{2} / \rho
$$

and (13) gives

$$
u_{1+}(\tau)=\sigma \operatorname{sat}\left(f(\xi)-k_{2} \bar{v}_{\tau}\right) .
$$

It suffices to show

$$
f(\xi)-k_{2} \bar{v}_{\tau}=\bar{v}_{\tau}^{2} / \rho<\bar{u}_{1}
$$

to prove $u_{1+}(\tau)=u_{1-}(\tau)$. Indeed, the equation holds from the definition of $\bar{v}_{\tau}$ and the inequality from the assumption directly.
From above, we have proven $u_{1-}(0)=0$ and $u_{1+}(\tau)=$ $u_{1-}(\tau)$, i.e., the controller $(7)$ is continuous over $[0, \infty)$.

Next, notice that the control law (10) is monotonic $(b=$ $0)$ and $v(0)=0$, then $y_{1}$ moves from $y_{1}(0)=\operatorname{ref}_{1}$ to $y_{1}(\tau)=\operatorname{ref}_{1}+\sigma r$ monotonically. Therefore, the constraint (4) is satisfied.

Finally, by noting $v_{\tau}^{2} \leq \rho \bar{u}_{1}$ from the second equation of (11), we have

$$
\left|u_{1-}(t)\right|=|M a t| \leq|M a \tau|=v_{\tau}^{2} / \rho \leq \bar{u}_{1}
$$

which proves the constraint (3). The proof is thus complete.

In Theorem 3.1, we assume $|\delta| \geq 2 r$ (or, $|\xi| \geq r$ ) which means that the two manifold $\mathbf{S}_{1}$ and $\mathbf{S}_{2}$ do not overlap. When $|\delta|<2 r$ (or, $|\xi|<r$ ), the controller in Theorem 3.1 may not work directly because a small $|\xi|$ gives a small $f(\xi)$ and hence a small $\bar{v}_{\tau}$ which implies a large $\tau$. In particular, when $|\delta|=r$, we have $\xi=0$ and $\tau=\infty$. However, $\tau$ should be small enough such that $t_{S}-\tau$ is sufficent for the previous PTOS to settle down. Nevertheless, the controller in Theorem 3.1 still works with a slight modification by resetting a smaller $r=|\delta| / 2$. With this modification, we will show that there is an upper boundary for $\tau$, which is independent of $r, \operatorname{ref}_{1}$, and $\operatorname{ref}_{2}$. The result is given below.

Corollary 3.1: For a given $\bar{r}$ and any $r \in(0, \bar{r}]$, the preview control time $\tau$ set in Theorem 3.1-(ii) has an upper boundary, i.e., $\tau \leq \bar{\tau}$, where $\bar{\tau}$ is independent of $r$, ref $_{1}$, and $\mathrm{ref}_{2}$. In particular,

$$
\begin{array}{r}
\bar{\tau}=3 / \min \left\{\sqrt{\bar{\rho} \bar{u}_{1} / \bar{r}}, \sqrt{\left(\bar{\rho} k_{2} / 2\right)^{2}+\bar{\rho} \bar{k}_{1}}-\bar{\rho} k_{2} / 2\right\} \\
\bar{\rho}=3 /(2 M), \bar{k}_{1}=\min \left\{k_{1}, \bar{u}_{1} / \bar{r}\right\} .
\end{array}
$$

Proof: In Theorem 3.1, we assume $|\delta| \geq 2 r$ which implies $|\xi| \geq r$. From the definitions of $f$ and $\bar{k}_{1}$, we have

$$
f(\xi) / r \geq f(r) / r \geq \bar{k}_{1} .
$$

Since the equations (11) give $\tau=3 / \sqrt{(\bar{\rho} / r) \operatorname{sat}\left(\bar{v}_{\tau}^{2} /(\bar{\rho} r)\right)}$, it suffices to prove

$$
\begin{array}{r}
\sqrt{(\bar{\rho} / r) \operatorname{sat}\left(\bar{v}_{\tau}^{2} /(\bar{\rho} r)\right)} \geq \\
\min \left\{\sqrt{\bar{\rho} \bar{u}_{1} / \bar{r}}, \sqrt{\left(\bar{\rho} k_{2} / 2\right)^{2}+\bar{\rho} \bar{k}_{1}}-\bar{\rho} k_{2} / 2\right\} .
\end{array}
$$

If $\bar{v}_{\tau}^{2} /(\bar{\rho} r) \geq \bar{u}_{1}$, the inequality (16) holds obviously. Otherwise, we have

$$
\text { lhs }=\bar{v}_{\tau} / r=\sqrt{\left(\bar{\rho} k_{2} / 2\right)^{2}+\bar{\rho} f(\xi) / r}-\bar{\rho} k_{2} / 2 \geq \mathrm{rhs}
$$

using (15). The proof is thus complete.

\section{B. Solvability of II-B}

The secondary actuator controller is a form of Composite Nonlinear Feedback (CNF) borrowed from [6]. Its control law is given by:

$$
u_{2}=u_{2 L}+u_{2 N}
$$

where $u_{2 L}$ is a linear feedback law which stabilizes the secondary actuator with a higher bandwidth than that of 
the primary, and $u_{2 N}$ is a nonlinear feedback law which improves the performance of the overall DSA system. The linear controller is given by standard state feedback gain,

$$
u_{2 L}=W x_{2},
$$

where $W=\left[\begin{array}{ll}w_{1} & w_{2}\end{array}\right]$ may be calculated by any linear control technique. The nonlinear feedback controller is given by:

$$
u_{2 N}=\gamma\left(\operatorname{ref}_{2}, y\right) H\left[\begin{array}{c}
p-\operatorname{ref}_{2} \\
v
\end{array}\right]
$$

where $H$ is chosen as:

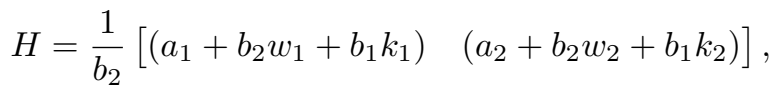

with constants $k_{1}$ and $k_{2}$ from (9), and the nonlinear function $\gamma(\cdot)$ is:

$$
\gamma\left(\operatorname{ref}_{2}, y\right)=e^{-\beta\left|\mathrm{ref}_{2}-y\right|}
$$

where $\beta$ is a tuning parameter.

Due to the proper choice of $H$ and $\gamma\left(\operatorname{ref}_{2}, y\right)$, the DSA closed-loop dynamics change from the primary to the secondary actuator control loop as the system approaches the reference point. This transition results in an improved performance inasmuch as the secondary actuator is designed to have a high bandwidth and a small damping ratio, allowing it to compensate the overshoot generated by the primary actuator [6]. Therefore, for the DSA system in (1) with the primary actuator under the control law (7), the secondary actuator under the nonlinear control law (17) is able to compensate for the error generated by the primary actuator under constraint (6), i.e., problem II-B is solved.

\section{EXPERIMENTAL RESULTS}

The proposed control scheme was implemented in the experimental DSA setup in [6]. The system is comprised of a linear motor (LM) as the primary stage and a piezo actuator (PZT) as the secondary stage. The LM has a $0.5 \mathrm{~m}$ travel range and a $1 \mu \mathrm{m}$ resolution glass scale encoder. The PZT has a maximum travel range of $\pm 15 \mu \mathrm{m}$ and an integrated capacitive position sensor with $0.2 \mathrm{~nm}$ resolution to measure the relative displacement between the LM and the PZT. The resonance of the PZT is actively damped by its integrated control electronics.

In order to compensate for the friction present in the LM, a model-based friction compensator was employed [11]. Thus, the primary actuator is modeled as a double integrator and the DSA is fully described by the set of equations in (1). For this particular system $\bar{u}_{1}=1 \mathrm{~V}$ and $\bar{u}_{2}=5 \mathrm{~V}$, and the parameters $a_{1}, a_{2}, b_{1}$ and $b_{2}$ were identified experimentally and are given by

$$
\begin{array}{ll}
a_{1}=-10^{6}, & b_{1}=1.5 \times 10^{7}, \\
a_{2}=-1810, & b_{2}=3 \times 10^{6} .
\end{array}
$$

When working in its linear region, the PTOS control law becomes a linear feedback gain $K=\left[k_{1} k_{2}\right]$ which may be parameterized as,

$$
K=\frac{1}{b_{1}}\left[\left(2 \pi \omega_{1}\right)^{2} 4 \pi \omega_{1} \zeta_{1}\right]
$$

with $\omega_{1}$ and $\zeta_{1}$ the natural frequency and damping ratio of the primary actuator closed-loop system. By pushing $\omega_{1}=$ $30 \mathrm{~Hz}$, the PTOS linear region is given by $\left|\mathrm{ref}_{2}-p(t)\right| \leq$ $422 \mu \mathrm{m}$. Similarly, the PZT gain is calculated by choosing $\omega_{2}=300 \mathrm{~Hz}$. The gains are given by,

$$
\begin{aligned}
K & =10^{-3} \times\left[\begin{array}{ll}
2.4 & 0.0225
\end{array}\right], \\
W & =-\left[\begin{array}{ll}
0.8385 & 0.0005
\end{array}\right] \\
H & =-\left[\begin{array}{ll}
1.1602 & 0.001
\end{array}\right]
\end{aligned}
$$

In the nonlinear function (21) the free parameter is chosen as $\beta=0.001$.

In order to add robustness to the preview control strategy, the feedback/feedforward scheme in Fig. 2 was implemented. The preview control input $u_{1-}$ is applied to an internal reference model, from which the desired trajectory $\hat{x}_{1}$ is obtained. Then, this trajectory is tracked by applying the designed preview input as a feedforward reference and by stabilizing the system with a linear feedback gain $Q=$ $\left[\begin{array}{ll}q_{1} & q_{2}\end{array}\right]$, which may computed by a standard linear control technique.

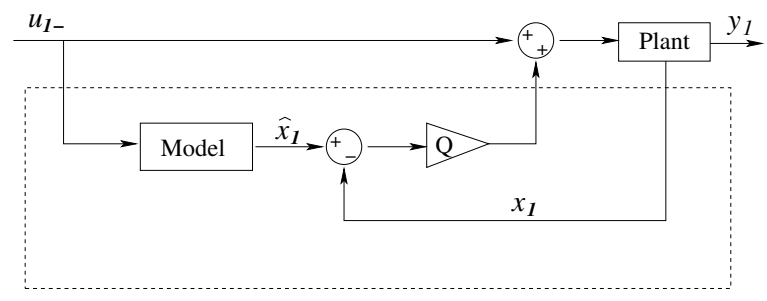

Fig. 2. Preview control strategy implemented in the primary actuator. A feedforward/feedback scheme is used in order to add robustness to the controller.

Three forms of DSA control strategy were compared in the experimental setup: (a) a conventional form of DSA control, where the primary actuator is tuned to have no overshoot [12]; (b) the nonlinear feedback control without preactuation, where the primary actuator is allowed to present some overshoot for improved performance [6]; and (c) the proposed preview control strategy. In order to implement controllers (a) and (b), different values of $\zeta_{1}$ in (23) were chosen accordingly. All controllers were implemented by a DSP system (dSPACE-DS1103) with the sampling frequency of $5 \mathrm{kHz}$, and settling time was defined as the time it takes for the total position output $y$ to enter and remain within $\pm 2 \mu \mathrm{m}$ relative to the setpoint.

Figures 3 and 4 show the step responses for references of $30 \mu \mathrm{m}$ and $100 \mu \mathrm{m}$, respectively. The top plot (a) shows the conventional control, the middle plot (b) shows the nonlinear feedback without pre-actuation, and the bottom plot (c) shows the proposed preview controller. In those plots the thick line is the total output of the system $(y)$, the dashed line is the primary actuator output $\left(y_{1}\right)$, and the dashdotted line is the secondary actuator output $\left(y_{2}\right)$. The $30 \mu \mathrm{m}$ $(100 \mu \mathrm{m})$ comparative improvement is of $83 \%$ (81\%) over a single stage actuator; $75 \%$ ( $70 \%$ ) over controller (a) and $70 \%$ ( 64\%) over controller (b). Fig. 5 shows the response of the three different controllers for the successive set point 


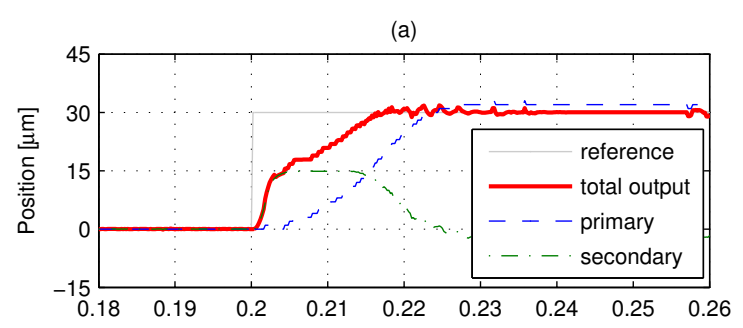

(b)

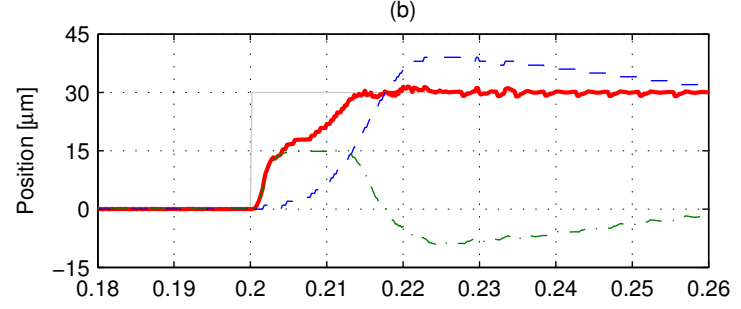

(c)

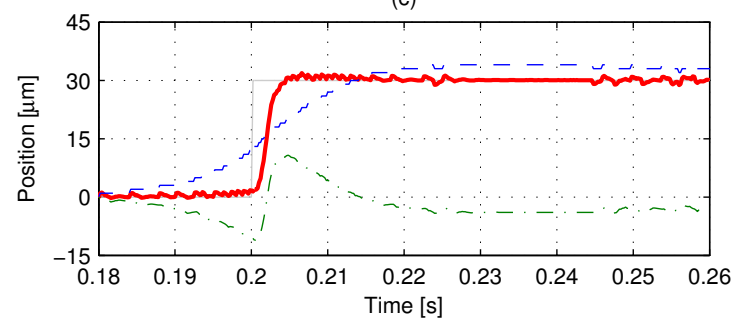

Fig. 3. Responses for a $30 \mu \mathrm{m}$ step reference, the proposed control design (c) has a settling time of $3.8 \mathrm{~ms}$ and a preview control time of $20.0 \mathrm{~ms}$.

scenario. These plots demonstrate the effectiveness of the of the proposed design.

\section{CONCLUSION}

A form of preview control for DSA systems was presented in this paper. Based on the information of future reference points, a control strategy was developed so that inputs were applied before the transition time interval. This control strategy takes full advantage of the redundancy of actuators enabling the DSA system to achieve an improved performance. Experimental results showed the effectiveness of the proposed approach which is able to significantly reduce the settling time of the overall DSA system.

\section{REFERENCES}

[1] K.W. Chan, W.H. Liao, and I.Y. Shen, "Precision positioning of hard disk drives using piezoelectric actuators with passive damping," IEEE/ASME Trans. Mech., vol. 13, no. 1, pp. 147-151, Feb. 2008.

[2] Bryson, A. E. and Ho, Y. C., Applied Optimal Control. New York: Hemisphere, 1975.

[3] M. Workman, "Adaptive Proximate Time-Optimal Control Servomechanisms," Ph.D. thesis, Stanford University, 1987.

[4] Z. Lin, M. Pachter, and S. Banda, "Toward improvement of tracking performance-nonlinear feedback for linear systems," Int. J. Control, vol. 70, pp. 1-11, 1998.

[5] B. M. Chen, T. H. Lee, K. Peng, and V. Venkataramanan, "Composite nonlinear feedback control for linear systems with input saturation: theory and an application," IEEE Trans. Automat. Contr., vol. 48, no. 3, pp. 427-439, Mar. 2003.

[6] J. Zheng, and M. Fu, "Nonlinear Feedback Control of a Dual-Stage Actuator System for Reduced Settling Time," IEEE Trans. Contr. Syst. Technol., vol. 16, no. 4, pp. 717-725, Jul. 2008.
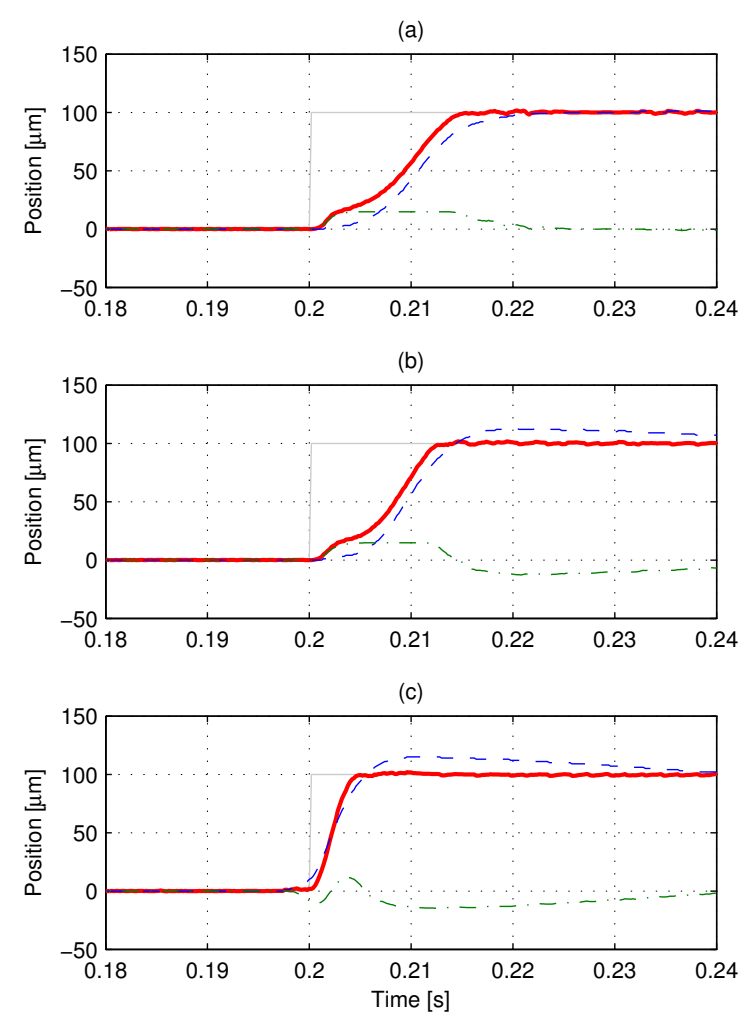

Fig. 4. Responses for a $100 \mu \mathrm{m}$ step reference, the proposed control design (c) has a settling time of $4.4 \mathrm{~ms}$ and a preview control time of $7.8 \mathrm{~ms}$.

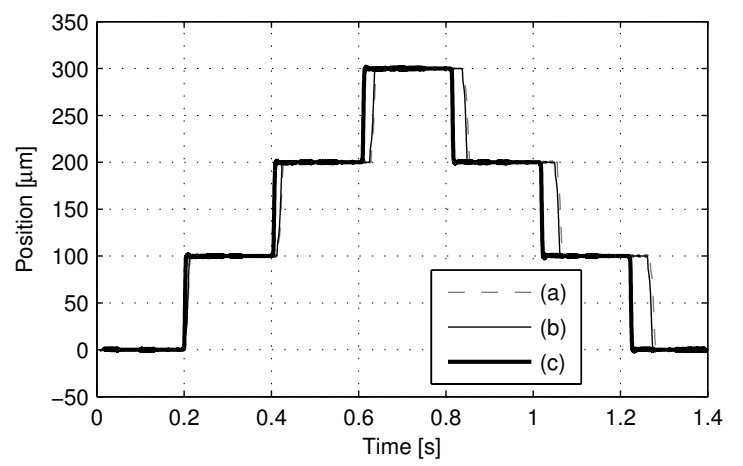

Fig. 5. Staircase response for the three different controllers. Here only the total output $y$ is depicted.

[7] A.T. Salton, Z. Chen, J. Zheng and M. Fu, "Preview Control of Dual-Stage Actuator Systems for Super Fast Transition Time," IEEE/ASME Trans. Mech. (99), pp. 1-6 [Online]. Avilable: http://ieeexplore.iee.org, DOI: 10.1109/TMECH.2010.2053851.

[8] M. Kobayashi, and R. Horowitz, "Track seek control for hard disk dual-stage servo systems," IEEE Trans. Magn., vol. 37, no. 2, pp. 949-954, Mar. 2001.

[9] B. Hredzak, G. Herrmann, and G. Guo, "A proximate-time-optimal control design and its application to a hard disk drive dual-stage actuator system," IEEE Trans. Magn., vol. 42, no. 6, pp. 1708-1715, Jun. 2006.

[10] D. Iamratanakul, D. and S. Devasia, "Minimum-Time/Energy, Output Transitions for Dual-Stage Systems," ASME J. Dynamic Systems, Measurement, and Control, vol. 131, pp. 1-8, Mar. 2009.

[11] H. Choi, B. Kim, I. Suh, and W. Chung, "Design and robust highspeed motion controller for a plant with actuator saturation," Journal of Dynamic systems, Measurement, and Control, vol. 122, pp. 535-541, Sep. 2000.

[12] S. Lee, and Y. Kim, "Minimum destructive interference design of dual stage control systems for hard disk drives," IEEE Trans. Contr. Syst. Technol., vo. 12, no.4, pp. 517-531, Jul. 2004. 